\title{
Evolution of Generalized Space Curve as a Function of Its Local Geometry
}

\author{
Nassar H. Abdel-All1, Samah G. Mohamed1, Mariam T. Al-Dossary² \\ ${ }^{1}$ Mathematics Department, Faculty of Science, Assiut University, Assiut, Egypt \\ ${ }^{2}$ Mathematics Department, Girls College of Science, University of Dammam, Dammam, KSA \\ Email: samah gaber2000@yahoo.com
}

Received 19 May 2014; revised 28 June 2014; accepted 11 July 2014

Copyright (C) 2014 by authors and Scientific Research Publishing Inc.

This work is licensed under the Creative Commons Attribution International License (CC BY).

http://creativecommons.org/licenses/by/4.0/

(c) (i) Open Access

\begin{abstract}
Kinematics of moving generalized curves in a $n$-dimensional Euclidean space is formulated in terms of intrinsic geometries. The evolution equations of the orthonormal frame and higher curvatures are obtained. The integrability conditions for the evolutions are given. Finally, applications in $R^{2}$ are given and plotted.
\end{abstract}

Keywords

Inelastic Curve, Curve Flow, Evolution of Curves

\section{Introduction}

The flow of a curve is called inelastic if the arclength of this curve is preserved. Inelastic curve flows have an importance in many applications such as engineering, computer vision [1] [2], computer animation [3] and even structural mechanics [4]. Physically, inelastic curve flows give rise to motion which no strain energy is induced. There exist such motions in many physical applications. G. S. Chirikjian and J. W. Burdick [5] studied applications of inelastic curve flows. M. Gage, R. S. Hamilton [6] and M. A. Grayson [7] investigated shrinking of closed plane curves to a circle via the heat equation. Also, D. Y. Kwon and F. C. Park [8] [9] derived the evolution equation for an inelastic plane and space curve. Latifi et al. [10] studied inextensible flows of curves in Minkowski 3-space.

The connection between integrable systems and differential geometry of curves and surfaces has been important topic of intense research [11] [12]. Goldstein and Petrich [13] showed that the celebrated mKdV equation naturally arises from inextensible motion of curves in Euclidean geometry. Nakayama, Segur and Wadati [14] set up a correspondence between the mKdV hierarchy and inextensible motions of plane curves in Euclidean geometry. Integrable systems satisfied by the curvatures of curves under inextensible motions in projective 
geometries are identified in [15]. Inextensible flows of curves in Galilean space are investigated in [16].

In this paper, we shall present a general formulation of evolving generalized curves in $R^{n}$. The outline of this paper is as follows: In Section 2, we give the local differential geometry of curves in $R^{n}$. In Sections 3 and 4 , we describe the motion of generalized curves in $R^{n}$. In Section 5 , the integrability conditions for the considered model are obtained. In Section 6 , we specialized the motion of curves $R^{n}$ to motion of plane curves (curves in $R^{2}$ ). Finally, Section 7 is devoted to conclusion.

\section{Geometric Preliminaries}

A generalized curve in a $n$-dimensional Euclidean space $R^{n}$ can be regarded as a Riemannian submanifold of dimension 1 in $R^{n}$ [17].

Definition 1 A differentiable manifold of dimension 1 immersed in $R^{n}$ is a topological hausdorff space $M$ with a differentiable structure $\left(I_{\beta}, \phi_{\beta}\right)$ with dimension one, where $I_{\beta}$ is an open interval in $R$ and $\phi_{\beta}$ is a diffeomorphism mapping:

and $\beta$ belongs to some index set $\Lambda$.

$$
\phi_{\beta}: I_{\beta} \rightarrow R^{n},
$$

Definition 2 A Generalized curve $C$ in $R^{n}$ is an image of a diffomorphism $\phi: I \rightarrow R^{n}$, where $I$ is an open interval of $R$. The representation of $C$ in $R^{n}$ is given by

$$
u \in I \rightarrow \phi(u)=\left(x_{1}(u), x_{2}(u), \cdots, x_{n}(u)\right) \in R^{n},
$$

where $u$ is called the parameter of the curve $C$.

The representation (1.1) is called the regular parametric representation of $C$ in $R^{n}$, when

$$
\frac{\mathrm{d} \phi}{\mathrm{d} u} \neq 0, \quad u \in I
$$

Also $\phi$ represents an immersion in $R^{n}$ if $\left|\frac{\mathrm{d} \phi}{\mathrm{d} u}\right|=1$, the parameter $u$, in this case, is called the arclength parameter and is denoted by $s, \phi(s)$ is called arclength parametrization.

\section{Frenet Frame}

A Frenet frame is a moving reference frame of $n$ orthonormal vectors $e_{i}(s)$ which are used to describe the curve locally at each point $\phi(s)$. It is the main tool in differential geometric treatment of curves as it is far easier and more natural to describe the local properties (e.g. curvature and torsion) in terms of local reference system than using a global one like the Euclidean coordinates.

Give a curve $\phi$ in $R^{n}$ which is regular of order $n$. The Frenet frame for the curve is the set of orthonormal vectors (Frenet vectors) $\mathfrak{I}=\left\{e_{1}(s), e_{2}(s), \cdots, e_{n}(s)\right\}$, and they are constructed from the derivatives of $\left\{\phi(s), \phi^{\prime}(s), \phi^{\prime \prime}(s), \cdots, \phi^{(n)}(s)\right\}$, which are linearly independent vectors, $\left(\phi^{\prime}(s)=\frac{\mathrm{d}}{\mathrm{d} s}\right)$.

Using the Gram-Schmidt orthogonalization algorithm which convert linearly independent vectors $\left\{\phi^{\prime}(s), \phi^{\prime \prime}(s), \cdots, \phi^{(n)}(s)\right\}$ into the orthonormal one $\left\{e_{1}(s), e_{2}(s), \cdots, e_{n}(s)\right\}$ as follows:

$$
e_{1}(s)=\phi^{\prime}(s), e_{2}(s)=\frac{W_{2}}{\left|W_{2}\right|}, \cdots, e_{i}(s)=\frac{W_{i}}{\left|W_{i}\right|}, i=2, \cdots, n,
$$

where

$$
W_{2}(s)=\phi^{\prime \prime}(s)-\left\langle\phi^{\prime \prime}(s), e_{1}(s)\right\rangle e_{1}(s), \cdots, W_{i}(s)=\phi^{(i)}(s)-\sum_{k=1, k<i}^{n-1}\left\langle\phi^{(i)}(s), e_{k}(s)\right\rangle e_{k}(s) .
$$

By this way, one obtain an orthonormal $n$-tuple of vectors at $\phi(s)$, called the Frenet $n$-frame associated with the generalized curve at the point $\phi(s)$, with $e_{i}(s)$ is of class $C^{k-i}$ if $\phi(s) \in C^{k}$. The derivatives of the frenet $n$-frame at $\phi(s)$ satisfy the following Frenet formulas: 


$$
e_{1}^{\prime}=k_{1} e_{2}, e_{2}^{\prime}=-k_{1} e_{1}+k_{2} e_{3}, \cdots, e_{j}^{\prime}=-k_{j-1} e_{j-1}+k_{j} e_{j+1},
$$

where $j=1,2, \cdots, n, k_{0}=k_{n}=0$. These equations can be written in a matrix form:

$$
\frac{\mathrm{d} E}{\mathrm{~d} s}=Q \cdot E
$$

where

$$
E=\left(e_{1}, e_{2}, \cdots, e_{n}\right)^{t}, \quad Q=\left(\begin{array}{ccccccc}
0 & k_{1} & 0 & 0 & \cdots & 0 & 0 \\
-k_{1} & 0 & k_{2} & 0 & \cdots & 0 & 0 \\
0 & -k_{2} & 0 & k_{3} & \cdots & 0 & 0 \\
\vdots & \vdots & \vdots & \vdots & \vdots & \vdots & \vdots \\
0 & 0 & 0 & \cdots & -k_{n-2} & 0 & k_{n-1} \\
0 & 0 & 0 & \cdots & 0 & -k_{n-1} & 0
\end{array}\right),
$$

and $k_{1}(s), \cdots, k_{j}(s), \cdots, k_{n-1}(s)$ are higher curvature functions or Euclidean curvatures of the curve. The $m$-th Euclidean curvature $k_{m}$ gives the speed of rotation of the osculation $m$-plane around the osculating $(m-1)$-plane.

\section{Dynamics of Curves in $R^{n}$}

Consider a smooth curve in $n$-dimension space. Assume that $u$ is the parameter along the curve in $R^{n}$. Let $r(u, t)$ denotes the position vector of a point on the curve at time $t$. The metric on the curve is:

$$
g(u, t)=\left\langle\frac{\partial r}{\partial u}, \frac{\partial r}{\partial u}\right\rangle
$$

The arclength along the curve is given by:

$$
s(u, t)=\int_{0}^{u} \sqrt{g(\sigma, t)} \mathrm{d} \sigma, \quad \frac{\partial}{\partial s}=\frac{1}{\sqrt{g}} \frac{\partial}{\partial u},
$$

we use $\{u, t\}$ as coordinates of a point on the curve. At $r(u, t)$, consider the orthonormal frame $\mathfrak{I}=\left\{e_{1}, e_{2}, \cdots, e_{n}\right\}$ such that $e_{1}$ is the tangent vector and $e_{2}, e_{3}, \cdots, e_{n}$ denote the normal vectors at any point on the curve.

Dynamics of the curve in $R^{n}$ (motion of a point on the curve) can be specified by the form:

$$
\dot{r}=\frac{\mathrm{d} r}{\mathrm{~d} t}=\sum_{j=1}^{n} v_{j} e_{j},
$$

where $v_{j}$ are the velocities along the frame $e_{j}$. Consider a local motion that is the velocities $v_{j}$ depend only on the local values of the curvatures $\left\{k_{1}, k_{2}, \cdots, k_{j}, \cdots, k_{n-1}\right\}$.

Lemma 1 The evolution equation for the metric $g$ is given by:

$$
\dot{g}=2 g\left(\frac{\partial v_{1}}{\partial s}-k_{1} v_{2}\right) \text {. }
$$

Proof 1 Take the $t$ derivative of (1.5) and $s$ derivative of (1.7), and since $\frac{\partial}{\partial u}, \frac{\partial}{\partial t}$ commute, then we have:

$$
\dot{g}=\frac{\partial g}{\partial t}=2\left\langle\frac{\partial r}{\partial u}, \frac{\partial}{\partial t} \frac{\partial r}{\partial u}\right\rangle=2 g\left\langle\frac{\partial r}{\partial s}, \frac{\partial}{\partial s} \frac{\partial r}{\partial t}\right\rangle=2 g\left\langle e_{1},\left(\sum_{j=1}^{n} \frac{\partial v_{j}}{\partial s} e_{j}+\sum_{j=1}^{n} v_{j} \frac{\partial e_{j}}{\partial s}\right)\right\rangle
$$

Using (1.2), then we have

$$
\dot{g}=2 g\left\langle e_{1}, \lambda e_{1}+\sum_{j=2}^{n} A_{j} e_{j}\right\rangle
$$


where,

$$
\begin{aligned}
& \lambda=\left(\frac{\partial v_{1}}{\partial s}-k_{1} v_{2}\right), \\
& A_{j}=v_{j, s}+k_{j-1} v_{j-1}-k_{j} v_{j+1}, \\
& j=2,3, \cdots, n, \quad k_{0}=k_{n}=0 .
\end{aligned}
$$

Then

Hence the lemma holds.

$$
\dot{g}=2 g \lambda
$$

Lemma 2 For a simple closed curve, the evolution of the length of the curve is given by:

$$
\frac{\partial L}{\partial t}=\int_{0}^{u}\left(\frac{\partial v_{1}}{\partial s}-k_{1} v_{2}\right) \mathrm{d} \sigma, u \in[0, L]
$$

Proof 2 From the definition of the length $L$, we have

$$
\frac{\partial L}{\partial t}=\frac{\partial s}{\partial t}=\int_{0}^{u} \frac{\partial}{\partial t}(\sqrt{g}) \mathrm{d} \sigma=\int_{0}^{u} \frac{\dot{g}}{2 \sqrt{g}} \mathrm{~d} \sigma .
$$

Substitute from (1.8) into (1.10), then the lemma holds.

\section{Main Results}

Definition 3 An inelastic curve is a curve whose length is preserved, i.e., it doesn't evolve in time.

$$
\frac{\partial s}{\partial t}=0, \text { i.e., } \quad g_{t}=\dot{g}=0 .
$$

The necessary and sufficient conditions for inelastic flow are then given by the following theorem:

Theorem 1 The flow of the curve is inelastic if and only if $\frac{\partial v_{1}}{\partial s}=k_{1} v_{2}$.

Proof $3(\Rightarrow)$ Assume that the curve is inelastic.

From (1.6), the variation of the arclength is

$$
\dot{s}=\frac{\partial s}{\partial t}=\int_{0}^{u} \frac{g_{t}}{2 \sqrt{g}} \mathrm{~d} \sigma
$$

Substitute from (1.8) into (1.12), then

$$
\dot{s}=\int_{0}^{u}\left(\frac{\partial v_{1}}{\partial s}-k_{1} v_{2}\right) \mathrm{d} \sigma
$$

Since the curve is inelastic, so $\dot{s}=0$, hence

$$
\frac{\partial v_{1}}{\partial s}=k_{1} v_{2}
$$

$(\Leftarrow)$ Assume that $\frac{\partial v_{1}}{\partial s}=k_{1} v_{2}$. Substitute from this equation into (1.8), so $g_{t}=0$, then $\dot{s}=0$, this means that the arclength of the curve is preserved, hence the curve is inelastic.

Theorem 2 Consider an elastic curve $r(u, t)$. For the curve flow $\frac{\mathrm{d} r}{\mathrm{~d} t}=\sum_{j=1}^{n} v_{j} e_{j}$, then

1) The evolution for the frame $E=\left(e_{1}, e_{2}, \cdots, e_{n}\right)^{t}$, can be given in a matrix form:

$$
E_{t}=M \cdot E,
$$

where $M$ is the evolution matrix and it takes the form: 


$$
\boldsymbol{M}=\left(\begin{array}{ccccc}
0 & M_{12} & M_{13} & \cdots & M_{1 n} \\
-M_{12} & 0 & M_{23} & \cdots & M_{2 n} \\
-M_{13} & -M_{23} & 0 & \cdots & M_{3 n} \\
\vdots & \vdots & \vdots & \vdots & \vdots \\
-M_{1(n-1)} & -M_{2(n-1)} & -M_{3(n-1)} & \cdots & M_{(n-1) n} \\
-M_{1 n} & -M_{2 n} & -M_{3 n} & \cdots & 0
\end{array}\right),
$$

where the elements of the matrix $M$ are given explicitly by:

$$
\begin{aligned}
& M_{1 j}=A_{j}=v_{j, s}+k_{j-1} v_{j-1}-k_{j} v_{j+1}, \\
& j=2,3, \cdots, n . \\
& M_{\alpha \mu}=\frac{1}{k_{\mu-\alpha}}\left(M_{(\alpha-1) \mu, s}+k_{\mu-1} M_{(\alpha-1)(\mu-1)}-k_{\mu} M_{(\alpha-1)(\mu+1)}+k_{\alpha-2} M_{(\alpha-2) \mu}\right), \\
& \alpha=2, \cdots, n-1, \\
& \alpha<\mu \leq n, k_{0}=k_{n}=0 .
\end{aligned}
$$

2) The evolution equations for the curvatures take the form:

$$
\begin{aligned}
& k_{1, t}=M_{12, s}-k_{1} \lambda-k_{2} M_{13}, \\
& k_{\alpha, t}=M_{\alpha \mu, s}-k_{\alpha} \lambda+k_{\alpha-1} M_{(\alpha-1) \mu}-k_{\alpha+1} M_{\alpha(\mu+1)} .
\end{aligned}
$$

Proof 4 Consider the elastic curve $r(u, t)$ i.e., $(\dot{g} \neq 0)$. Take the $u$ derivative of (1.7), then we have:

$$
r_{t u}=\sqrt{g} r_{t s}=\sqrt{g}\left(\lambda e_{1}+\sum_{j=2}^{n} A_{j} e_{j}\right)
$$

Since $r_{u}=\sqrt{g} r_{s}=\sqrt{g} e_{1}$, take the $t$ derivative of this equation, then we have

$$
r_{u t}=\sqrt{g}\left(\frac{g_{t}}{2 g} e_{1}+e_{1, t}\right)
$$

Since

$$
r_{u t}=r_{t u}
$$

Substitute from (1.9), (1.16) and (1.17) into (1.18), then we have

$$
e_{1, t}=\sum_{j=2}^{n} A_{j} e_{j} .
$$

Take the $u$ derivative of (1.19), then we have:

$$
e_{1, t u}=\sqrt{g}\left(\left(-k_{1} A_{2}\right) e_{1}+\left(A_{2, s}-k_{2} A_{3}\right) e_{2}+\sum_{j=3}^{n}\left(A_{j, s}+k_{j-1} A_{j-1}-k_{j} A_{j+1}\right) e_{j}\right) .
$$

Since $e_{1, u}=\sqrt{g} e_{1, s}=\sqrt{g}\left(k_{1} e_{2}\right)$, take the $t$ derivative of this equation, then we have

$$
e_{1, u t}=\sqrt{g}\left(\left(\frac{g_{t}}{2 g} k_{1}+k_{1, t}\right) e_{2}+k_{1} e_{2, t}\right) \text {. }
$$

Since

$$
e_{1, u t}=e_{1, t u} \text {. }
$$

Substitute from (1.20) and (1.21) into (1.22), then we have 


$$
\begin{aligned}
& k_{1, t}=A_{2, s}-k_{1} \lambda-k_{2} A_{3}, \\
& e_{2, t}=-A_{2} e_{1}+\sum_{j=3}^{n} B_{j} e_{j}, \\
& B_{j}=\frac{1}{k_{1}}\left(A_{j, s}+k_{j-1} A_{j-1}-k_{j} A_{j+1}\right), \quad j=3, \cdots, n .
\end{aligned}
$$

Since $e_{2, u}=\sqrt{g} e_{2, s}=\sqrt{g}\left(-k_{1} e_{1}+k_{2} e_{3}\right)$, take the $t$ derivative of this equation, then we have

$$
e_{2, u t}=\sqrt{g}\left(-\left(k_{1} \lambda+k_{1, t}\right) e_{1}-\left(k_{1} A_{2}\right) e_{2}+\left(-k_{1} A_{3}+k_{2} \lambda+k_{2, t}\right) e_{3}+k_{2} e_{3, t}-k_{1} \sum_{j=4}^{n} A_{j} e_{j}\right) \text {. }
$$

Take the $u$ derivative of (1.23), then we have

$$
\begin{aligned}
e_{2, t u}= & \sqrt{g}\left(-A_{2, s} e_{1}-\left(k_{1} A_{2}+k_{2} B_{3}\right) e_{2}+\left(B_{3, s}-k_{3} B_{4}\right) e_{3}+\left(B_{4, s}+k_{3} B_{3}-k_{4} B_{5}\right) e_{4}+\cdots\right. \\
& \left.+\left(B_{n-1, s}+k_{n-2} B_{n-2}-k_{n-1} B_{n}\right) e_{n-1}+\left(B_{n, s}+k_{n-1} B_{n-1}\right) e_{n}\right) .
\end{aligned}
$$

Since

$$
e_{2, u t}=e_{2, t u} \text {. }
$$

Substitute from (1.24) and (1.25) into (1.26), and use the first equation of (1.23), then we have

$$
\begin{aligned}
& k_{2, t}=B_{3, s}-k_{2} \lambda-k_{3} B_{4}+k_{1} A_{3}, \\
& e_{3, t}=-A_{3} e_{1}-B_{3} e_{2}+\sum_{j=4}^{n} C_{j} e_{j}, \\
& C_{j}=\frac{1}{k_{2}}\left(B_{j, s}+k_{1} A_{j}+k_{j-1} B_{j-1}-k_{j} B_{j+1}\right), \quad j=4, \cdots, n .
\end{aligned}
$$

Take the $u$ derivative of the second equation of (1.27), then we have

$$
\begin{aligned}
e_{3, t u}= & \sqrt{g}\left(\left(-A_{3, s}+k_{1} B_{3}\right) e_{1}-\left(B_{3, s}+k_{1} A_{3}\right) e_{2}-\left(k_{2} B_{3}+k_{3} C_{4}\right) e_{3}+\left(C_{4, s}-k_{4} C_{5}\right) e_{4}+\left(C_{5, s}+k_{4} C_{4}-k_{5} C_{6}\right) e_{5}+\cdots\right. \\
& \left.+\left(C_{n-1, s}+k_{n-2} C_{n-2}-k_{n-1} C_{n}\right) e_{n-1}+\left(C_{n, s}+k_{n-1} C_{n-1}\right) e_{n}\right) .
\end{aligned}
$$

Since $e_{3, u}=\sqrt{g} e_{3, s}=\sqrt{g}\left(-k_{2} e_{2}+k_{3} e_{4}\right)$, take the $t$ derivative of this equation and use the first equation of (1.27), then we have

$$
e_{3, u t}=\sqrt{g}\left(k_{2} A_{2} e_{1}+\left(-B_{3, s}+k_{3} B_{4}-k_{1} A_{3}\right) e_{2}-k_{2} B_{3} e_{3}+\left(k_{3} \lambda+k_{3, t}-k_{2} B_{4}\right) e_{4}+k_{3} e_{4, t}-k_{2} \sum_{j=5}^{n} B_{j} e_{j}\right) .
$$

Since

$$
e_{3, u t}=e_{3, t u} \text {. }
$$

Substitute from (1.28) and (1.29) into (1.30), then we have

$$
\begin{aligned}
& k_{3, t}=C_{4, s}+k_{2} B_{4}-k_{3} \lambda-k_{4} C_{5}, \\
& e_{4, t}=-A_{4} e_{1}-B_{4} e_{2}-C_{4} e_{3}+\sum_{j=5}^{n} D_{j} e_{j}, \\
& D_{j}=\frac{1}{k_{3}}\left(C_{j, s}+k_{2} B_{j}+k_{j-1} C_{j-1}-k_{j} C_{j+1}\right), j=5,6, \cdots, n .
\end{aligned}
$$

By using the mathematical induction, we can extend the previous results to $n$-dimensional space as follows:

$$
E_{t}=M \cdot E,
$$


where $E=\left(e_{1}, e_{2}, \cdots, e_{n}\right)^{t}$, and

$$
\boldsymbol{M}=\left(\begin{array}{ccccccc}
0 & A_{2} & A_{3} & A_{4} & A_{5} & \cdots & A_{n} \\
-A_{2} & 0 & B_{3} & B_{4} & B_{5} & \cdots & B_{n} \\
-A_{3} & -B_{3} & 0 & C_{4} & C_{5} & \cdots & C_{n} \\
-A_{4} & -B_{4} & -C_{4} & 0 & D_{5} & \cdots & D_{n} \\
\vdots & \vdots & \vdots & \vdots & \vdots & \vdots & \vdots \\
-A_{n} & -B_{n} & -C_{n} & -D_{n} & \cdots & \cdots & 0
\end{array}\right) .
$$

We can rewrite (1.9) and the third equation of (1.23), (1.27) and (1.31) as follows:

$$
\begin{array}{ll}
A_{j}=M_{1 j}, & 1<j \leq n, \\
B_{j}=M_{2 j}, & 2<j \leq n, \\
C_{j}=M_{3 j}, & 3<j \leq n, \\
D_{j}=M_{4 j}, & 4<j \leq n .
\end{array}
$$

Hence

$$
\begin{aligned}
& M_{1 j}=A_{j}=v_{j, s}+k_{j-1} v_{j-1}-k_{j} v_{j+1}, \quad j=2,3, \cdots, n \\
& M_{\alpha \mu}=\frac{1}{k_{\mu-\alpha}}\left(M_{(\alpha-1) \mu, s}+k_{\mu-1} M_{(\alpha-1)(\mu-1)}-k_{\mu} M_{(\alpha-1)(\mu+1)}+k_{\alpha-2} M_{(\alpha-2) \mu}\right), \\
& \alpha=2, \cdots, n-1, \quad \mu=3, \cdots, n, \quad \alpha<\mu, \\
& k_{0}=k_{n}=0 .
\end{aligned}
$$

So we can rewrite (1.32) in the following form:

$$
\boldsymbol{M}=\left(\begin{array}{ccccc}
0 & M_{12} & M_{13} & \cdots & M_{1 n} \\
-M_{12} & 0 & M_{23} & \cdots & M_{2 n} \\
-M_{13} & -M_{23} & 0 & \cdots & M_{3 n} \\
\vdots & \vdots & \vdots & \vdots & \vdots \\
-M_{1(n-1)} & -M_{2(n-1)} & -M_{3(n-1)} & \cdots & M_{(n-1) n} \\
-M_{1 n} & -M_{2 n} & -M_{3 n} & \cdots & 0
\end{array}\right)
$$

By using the mathematical induction, we can extend the results in the first equation of (1.23), (1.27) and (1.31) as follows:

$$
\begin{aligned}
& k_{1, t}=M_{12, s}-k_{1} \lambda-k_{2} M_{13}, \\
& k_{\alpha, t}=M_{\alpha \mu, s}-k_{\alpha} \lambda+k_{\alpha-1} M_{(\alpha-1) \mu}-k_{\alpha+1} M_{\alpha(\mu+1)},
\end{aligned}
$$

Hence the theorem holds.

Lemma 3 If the curve flow (1.7) is inelastic, then the evolution equations for curvatures (1.36) take the form:

$$
\begin{aligned}
& k_{1, t}=M_{12, s}-k_{2} M_{13}, \\
& k_{\alpha, t}=M_{\alpha \mu, s}+k_{\alpha-1} M_{(\alpha-1) \mu}-k_{\alpha+1} M_{\alpha(\mu+1)},
\end{aligned}
$$

Proof 5 If the curve flow (1.7) is inelastic, then

$$
g_{t}=0 \text {, i.e., } \lambda=0 \text {. }
$$

Then, substitute from (1.38) into (1.36), then the lemma holds.

\section{Integrability Conditions}

Theorem 3 The curve $r(u, t)$ is inelastic curve $\left(g_{t}=0\right)$ if and only if the integrability condition (sometimes 
called the zero curvature condition) is given by:

$$
Q_{t}-M_{s}+[Q, M]=0,
$$

where $[Q, M]=Q \cdot M-M \cdot Q$ is the Lie bracket.

Proof 6 Consider the Frenet frame $E=\left(e_{1}, e_{2}, \cdots, e_{n}\right)^{t}$, that satisfy (1.4) and (1.35). Since

$$
E_{u}=\sqrt{g} E_{s}=\sqrt{g} Q \cdot E .
$$

Take the $t$ derivative of (1.40) and use (1.13), then we have

$$
E_{u t}=\sqrt{g}\left(\frac{g_{t}}{2 g} Q+Q_{t}+Q \cdot M\right) \cdot E .
$$

Differentiating (1.13) with respect to $u$ and use (1.40), then

$$
E_{t u}=\sqrt{g}\left(M_{s}+M \cdot Q\right) \cdot E .
$$

From (1.41) and (1.42), then

$$
E_{u t}-E_{t u}=\sqrt{g}\left(\frac{g_{t}}{2 g} Q+Q_{t}-M_{s}+[Q, M]\right) \cdot E
$$

$(\Rightarrow)$ First, If the curve is inelastic, so $\left(g_{t}=0\right)$ and $u$ and $t$ commute, then $E_{u t}=E_{t u}$, hence

$$
Q_{t}-M_{s}+[Q, M]=0 \text {. }
$$

$(\Leftarrow)$ Second, Assume that the integrability condition (the zero curvature condition) is satisfied, then

$$
Q_{t}-M_{s}+[Q, M]=0
$$

From (1.4) and (1.35), we have

$$
[Q, M]=\left(\begin{array}{ccccc}
0 & k_{2} M_{13} & M_{13, s} & \cdots & M_{1 n, s} \\
-k_{2} M_{13} & 0 & -k_{1} M_{13}+k_{3} M_{24} & \cdots & M_{2 n, s} \\
\vdots & \vdots & \vdots & \cdots & \vdots \\
-M_{1 n-1, s} & -M_{2 n-1, s} & -M_{3 n-1, s} & \cdots & -k_{n-2} M_{n-2 n} \\
-M_{1 n, s} & -M_{2 n, s} & -M_{31 n, s} & \cdots & 0
\end{array}\right) .
$$

Differentiating (1.4) with respect to $t$ and (1.35) with respect to $s$ and use (1.36), then we have

$$
M_{s}-Q_{t}=\left(\begin{array}{ccccc}
0 & -k_{2} M_{13}+\lambda k_{1} & M_{13, s} & \cdots & M_{1 n, s} \\
-k_{2} M_{13}+\lambda k_{1} & 0 & -\lambda k_{2}-k_{1} M_{13}+k_{3} M_{24} & \cdots & M_{2 n, s} \\
\vdots & \vdots & \vdots & \vdots & \vdots \\
-M_{1(n-1), s} & -M_{2(n-1), s} & -M_{3(n-1), s} & \cdots & -\lambda k_{n-1}-k_{n-2} M_{(n-2) n} \\
-M_{1 n, s} & -M_{2 n, s} & -M_{3 n, s} & \cdots & 0
\end{array}\right) \text {. }
$$

Substitute from (1.45) and (1.46) into (1.44), then we have

$$
\left(\begin{array}{ccccc}
0 & \lambda k_{1} & 0 & \cdots & 0 \\
\lambda k_{1} & 0 & -\lambda k_{2} & \cdots & 0 \\
\vdots & \vdots & \vdots & \vdots & \vdots \\
0 & 0 & 0 & \cdots & -\lambda k_{n-1} \\
0 & 0 & 0 & \cdots & 0
\end{array}\right)=\left(\begin{array}{c}
0 \\
0 \\
\vdots \\
0 \\
0
\end{array}\right)
$$

Hence

$$
\lambda k_{1}=0, \lambda k_{2}=0, \cdots, \lambda k_{n-1}=0 .
$$

Since $k_{m} \neq 0$, for $m=1,2, \cdots, n-1$. Then $\lambda=0$. Hence $\dot{g}=0, g=$ constant i.e., the arclength is preserved. Hence the curve is inelastic. 
Theorem 4 In $n$-dimensional Euclidean space, consider inelastic curve $r(u, t)$. If the matrices $Q$ and $M$ are abelian, then the elements in the evolution matrix $M$ take the form:

$$
M_{(\alpha-1) \mu}=0, \quad \alpha=2,3, \cdots, n-1, \mu=\alpha+1 .
$$

Proof 7 Since the matrices $Q$ and $M$ are abelian, so $[Q, M]=0$, then the integrability condition (1.39) takes the form:

$$
M_{s}-Q_{t}=0
$$

Since the curve is inelastic, so $\lambda=0$, then

$$
M_{s}-Q_{t}=\left(\begin{array}{ccccc}
0 & k_{2} M_{13} & M_{13, s} & \cdots & M_{1 n, s} \\
-k_{2} M_{13} & 0 & -k_{1} M_{13}+k_{3} M_{24} & \cdots & M_{2 n, s} \\
\vdots & \vdots & \vdots & \vdots & \vdots \\
-M_{1(n-1), s} & -M_{2(n-1), s} & -M_{3(n-1), s} & \cdots & -k_{n-2} M_{(n-2) n} \\
-M_{1 n, s} & -M_{2 n, s} & -M_{3 n, s} & \cdots & 0
\end{array}\right) .
$$

Substitute from (1.49) into (1.48), then for $n=10$, we have

$$
M_{13}=M_{35}=M_{57}=M_{79}=0, M_{24}=M_{46}=M_{68}=M_{8(10)}=0,
$$

By using the mathematical induction, we can extend the previous results to $n$-dimensional space, then we have

$$
M_{(\alpha-1) \mu}=0, \quad \alpha=2,3, \cdots, n-1, \mu=\alpha+1
$$

\section{Applications}

Here we give some applications for time evolution equations for plane curve. We are in a position to derive time evolution of geometrical quantities. For $n=2$, and from (1.13), we have motion of the Frenet frame of the curve in the plane.

Lemma 4 In 2 -dimensional Euclidean space, consider an elastic curve $r(u, t)$. The time evolution equation for the frame $E=\left(e_{1}, e_{2}\right)^{t}$ is given by

$$
\left(\begin{array}{l}
e_{1} \\
e_{2}
\end{array}\right)_{t}=\left(\begin{array}{cc}
0 & M_{12} \\
-M_{12} & 0
\end{array}\right)\left(\begin{array}{l}
e_{1} \\
e_{2}
\end{array}\right)
$$

where,

$$
M_{12}=v_{2, s}+k_{1} v_{1} .
$$

Lemma 5 The time evolution equation for the curvature of the curve in $R^{2}$ is given explicitly by

$$
k_{1, t}=v_{2, s s}+k_{1}^{2} v_{2}+v_{1} k_{1, s}
$$

This equation represents a quasilinear parabolic partial differential equation (PDE). This result coincide with [18].

Example 1 If

$$
v_{1}=k_{1}^{2}, \quad v_{2}=k_{1, s s s} .
$$

Then (1.50) takes the form:

$$
k_{1, t}=k_{1, \text { ssss }}+k_{1}^{2} k_{1, \text { sss }}+k_{1}^{2} k_{1, s} .
$$

The solution of the PDE (1.51) is

$$
k_{1}(s, t)=\sqrt{10} \operatorname{sech}\left(c_{1}-\frac{4 s+t}{4 \sqrt{2}}\right),
$$


where $c_{1}$ is constant. The curvature $k_{1}(s, t)$ of the curve is plotted as a function of $s$ and $t$ (Figure 1(a)), and for different values of $t$, the curvature $k_{1}(s)$ is plotted (Figure 1(b)).

Example 2 If

$$
v_{1}=k_{1}^{2}, \quad v_{2}=-k_{1, s} .
$$

Then (1.50) takes the form:

$$
k_{1, t}=-k_{1, s s s} .
$$

The solution of the PDE (1.52) is

$$
k_{1}(s, t)=\frac{1}{\sqrt{\lambda}}\left(c_{1} \mathrm{e}^{\sqrt{\lambda}(s-\lambda t)}-c_{2} \mathrm{e}^{-\sqrt{\lambda}(s-\lambda t)}\right)+c_{3},
$$

where $c_{1}, c_{2}, c_{3}$ and $\lambda$ are constants. The curvature $k_{1}(s, t)$ of the curve is plotted as a function of $s$ and $t$ (Figure 2(a)), and for different values of $t$, the curvature $k_{1}(s)$ is plotted (Figure 2(b)).

\section{Example 3 If}

$$
v_{1}=k_{1}^{2}, \quad v_{2}=k_{1, s}
$$

Then (1.50) takes the form:

$$
k_{1, t}=k_{1, s s s}+2 k_{1}^{2} k_{1, s} .
$$

The solution of the PDE (1.53) is

$$
k_{1}(s, t)=\sqrt{3} \operatorname{sech}\left(c_{2}+c_{1} s+c_{1}^{3} t\right),
$$

where $c_{1}$ and $c_{2}$ are constants. The curvature $k_{1}(s, t)$ of the curve is plotted as a function of $s$ and $t$ (Figure 3(a)), and for different values of $t$, the curvature $k_{1}(s)$ is plotted (Figure 3(b)).

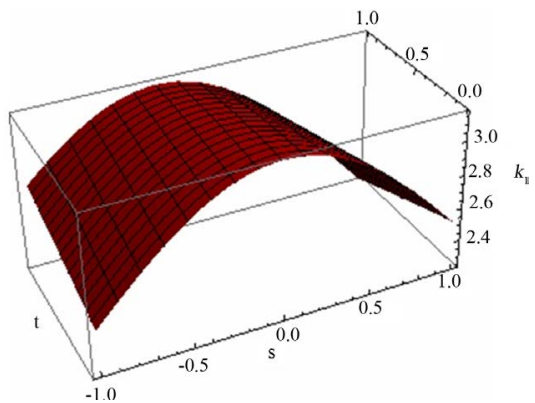

(a)

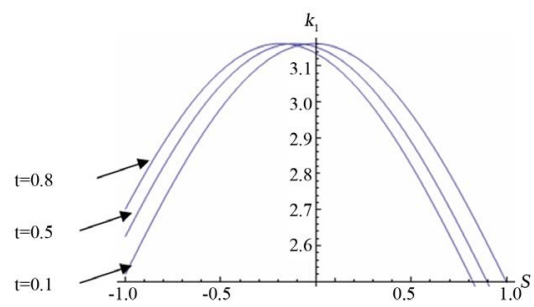

(b)

Figure 1. The curvature of the curve for $c_{1}=0.01, s \in[-1,1], t \in[0,1]$.

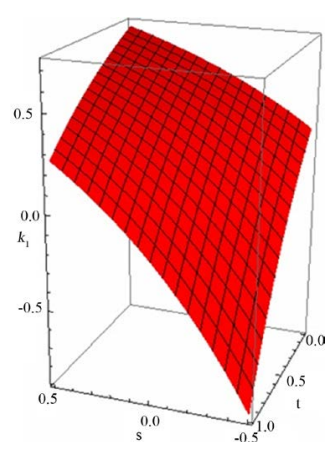

(a)

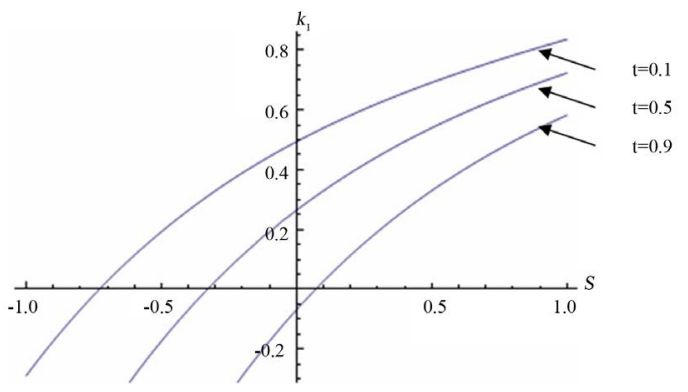

(b)

Figure 2. The curvature of the curve for $\lambda=1, c_{1}=0.04, c_{2}=0.4, c_{3}=0.9, s \in[-0.5,0.5], t \in[0,1]$. 


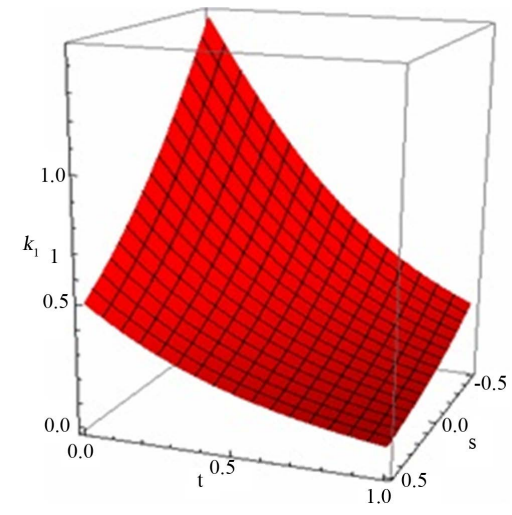

(a)

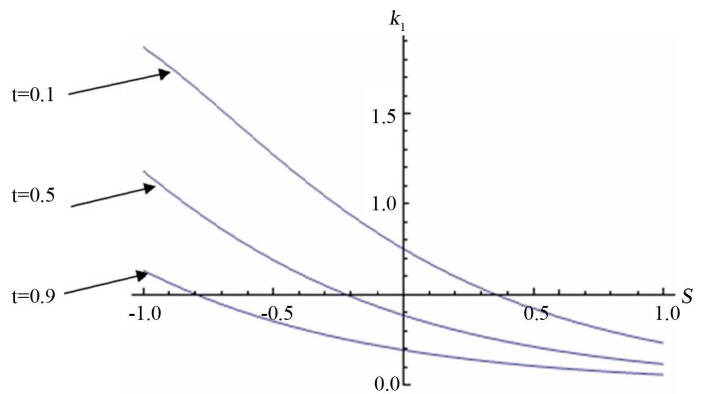

(b)

Figure 3. The curvature of the curve for $c_{1}=1.2, c_{2}=1.5, s \in[-0.5,0.5], t \in[0,1]$.

\section{Conclusion}

In this paper, we have discussed the motion of curves in $n$-dimension Euclidean space. We derived the evolution equations of the orthonormal frame and evolution equations for the higher curvatures. We get the integrability conditions for the evolutions. Moreover, we give some examples of motions of elastic curves in the plane.

\section{Acknowledgements}

The authors would like to thank the referee for helpful suggestions which improve the presentation of this work.

\section{References}

[1] Lu, H.Q., Todhunter, J.S. and Sze, T.W. (1993) Congruence Conditions for Nonplanar Developable Surfaces and Their Application to Surface Recognition. CVGIP, Image Understanding, 58, 265-285. http://dx.doi.org/10.1006/ciun.1993.1042

[2] Kass, M., Witkin, A. and Terzopoulos, D. (1987) Snakes: Active Contour Models. Proceedings of the 1st International Conference on Computer Vision (ICCV'87), London, June 1987, 259-268.

[3] Desbrun, M. and Cani, M.P. (1998) Active Implicit Surface for Animation. Proceedings of the Graphics Interface 1998 Conference, Vancouver, 18-20 June 1998.

[4] Unger, D.J. (1991) Developable Surfaces in Elastoplastic Fracture Mechanics. International Journal of Fracture, 50, 33-38. http://dx.doi.org/10.1007/BF00032160

[5] Chirikjian, G.S. and Burdick, J.W. (1990) Kinematics of Hyper-Redundant Manipulators. Proceeding of the ASME Mechanisms Conference, Vol. 25, Chicago, 16-19 September 1990, 391-396.

[6] Gage, M. and Hamilton, R.S. (1986) The Heat Equation Shrinking Convex Plane Curves. Journal of Differential Geometry, 23, 69-96.

[7] Grayson, M.A. (1987) The Heat Equation Shrinks Embedded Plane Curves to Round Points. Journal of Differential Geometry, 26, 223-370.

[8] Kwon, D.Y. and Park, F.C. (1999) Evolution of Inelastic Plane Curves. Applied Mathematics Letters, 12, 115-119. http://dx.doi.org/10.1016/S0893-9659(99)00088-9

[9] Kwon, D.Y., Park, F.C. and Chi, D.P. (2005) Inextensible Flows of Curves and Developable Surfaces. Applied Mathematics Letters, 18, 1156-1162. http://dx.doi.org/10.1016/j.aml.2005.02.004

[10] Latifi, D. and Razavi, A. (2008) Inextensible Flows of Curves in Minkowskian Space. Advanced Studies in Theoretical Physics, 2, 761-768.

[11] Bobenko, A., Sullivan, J., Schröder, P. and Ziegler, G. (2008) Discrete Differential Geometry. Oberwolfach Seminars, Vol. 38, X+341 p.

[12] Rogers, C. and Schief, W.K. (2002) Bäcklund and Darboux Transformations. Geometry and Modern Applications in Soliton Theory. Cambridge Texts in Applied Mathematics, Cambridge University Press, Cambridge.

[13] Goldstein, R.E. and Petrich, D.M. (1991) The Korteweg-de Vries Hierarchy as Dynamics of Closed Curves in the 
Plane. Physical Review Letters, 67, 3203-3206. http://dx.doi.org/10.1103/PhysRevLett.67.3203

[14] Nakayama, K., Segur, H. and Wadati, M. (1992) Integrability and the Motion of Curves. Physical Review Letters, 69, 2603-2606. http://dx.doi.org/10.1103/PhysRevLett.69.2603

[15] Li, Y.Y., Qu, C.Z. and Shu, S. (2010) Integrable Motions of Curves in Projective Geometries. Journal of Geometry and Physics, 60, 972-985. http://dx.doi.org/10.1016/j.geomphys.2010.03.001

[16] Öğrenmiş, A.O. and Yeneroğlu, M. (2010) Inextensible Curves in the Galilean Space. IJPS, 5, 1424-1427.

[17] Do Carmo, M.P. (1976) Differential Geometry of Curves and Surfaces. Englewood Cliffs, New Jersey.

[18] Nakayama, K. and Wadati, M. (1993) Motion of Curves in the Plane. Journal of the Physical Society of Japan, 62, 473-479. http://dx.doi.org/10.1143/JPSJ.62.473 
Scientific Research Publishing (SCIRP) is one of the largest Open Access journal publishers. It is currently publishing more than 200 open access, online, peer-reviewed journals covering a wide range of academic disciplines. SCIRP serves the worldwide academic communities and contributes to the progress and application of science with its publication.

Other selected journals from SCIRP are listed as below. Submit your manuscript to us via either submit@scirp.org or Online Submission Portal.
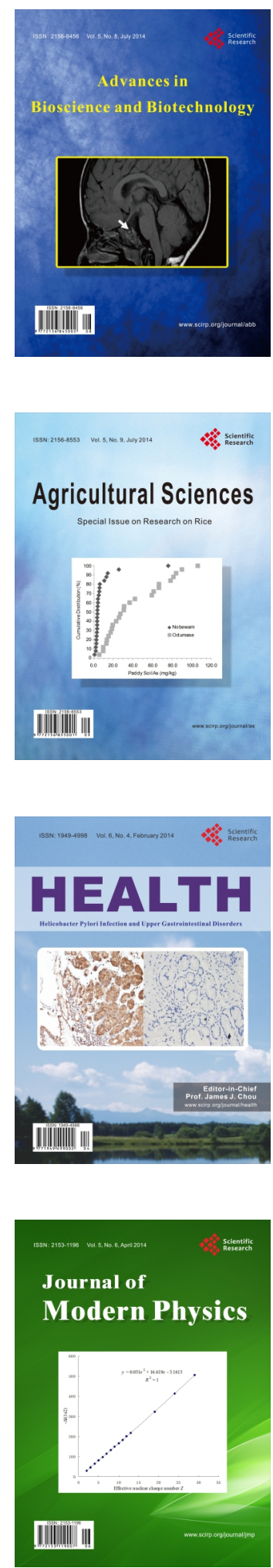
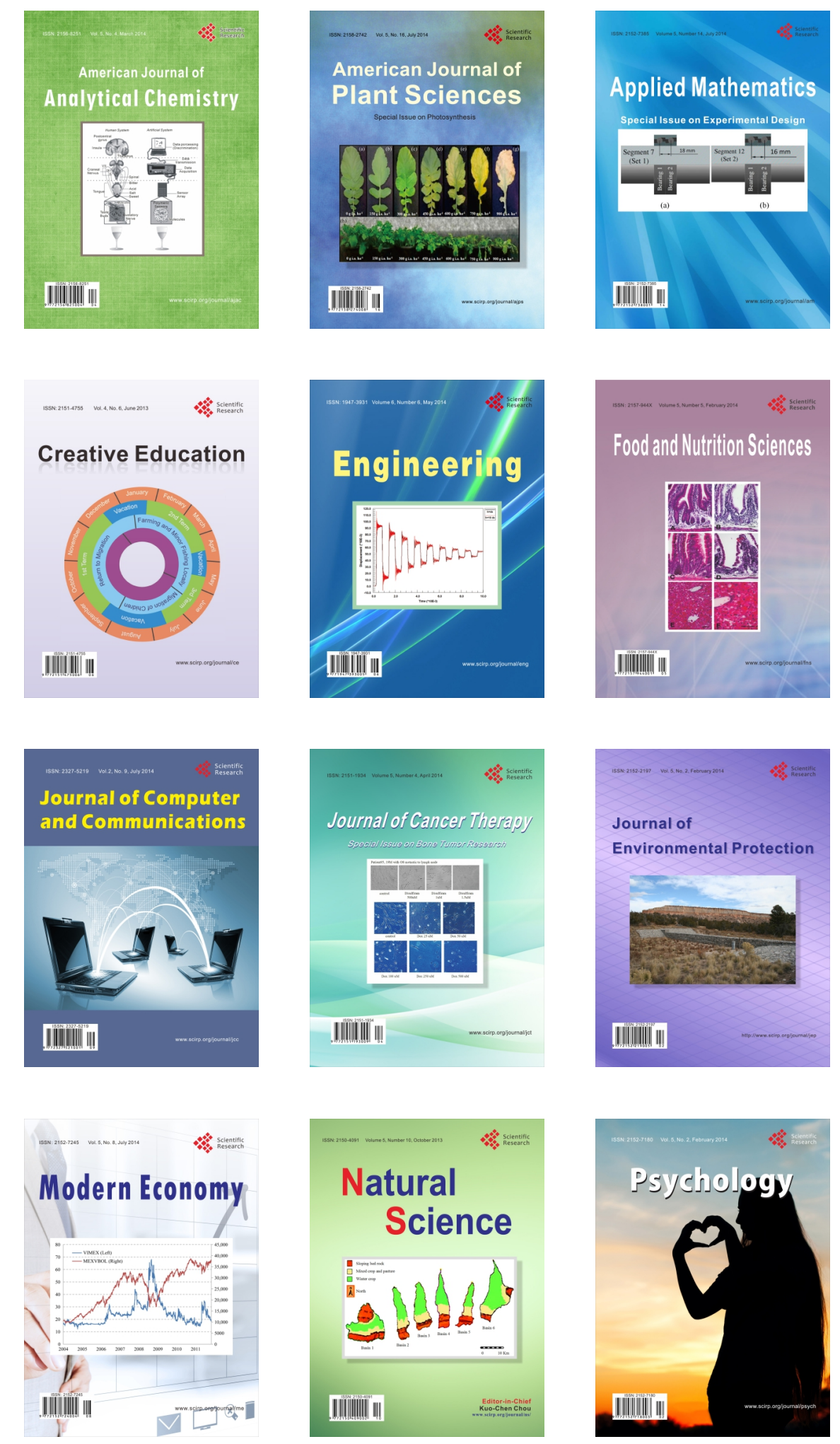